\title{
EREBEA
}

Revista de Humanidades

y Ciencias Sociales

Núm. 5 (2015), pp. 19-36

ISSN: 0214-0691

\section{En torno a Mahoma como Mesías UNA NUEVA MIRADA A LAS RAÍCES CRISTIANAS DEL ISLAM ${ }^{1}$}

\author{
Carlos A. Segovia \\ Saint Louis University - Madrid Campus, Spain
}

RESUMEN

Suele pensarse que el Corán despliega una doble y contradictoria actitud - a la vez favorable y desfavorable- hacia el cristianismo. Pero el Corán no contiene únicamente dos tipos de textos de esta índole, sino cuatro. Ahora bien, ¿cómo estudiar tales fórmulas? ¿Buscó Mahoma el respaldo de ciertos grupos cristianos distanciándose luego de éstos al rechazar ellos sus enseñanzas? El presente artículo plantea una hipótesis diferente, según la cual tales fórmulas dan testimonio del surgimiento gradual del islam entre 610 y 710 . Y explora asimismo el modo en que Jesús fue parcialmente sustituido entonces por Mahoma en calidad de nuevo mesías, analizando a tal fin diversos textos que sugieren que este último, al igual quizá que ya antes el profeta anónimo del Corán, fue identificado — como también Jesús - con el Hijo del Hombre de la tradición apocalíptica.

Palabras Clave

Corán; Cristianismo; Islam; Mahoma; Mesías; Apocalíptica; Hijo del Hombre.

Fecha de recepción: 24 de sept. de 2015 Fecha de aceptación: 30 de octubre de 2015
Abstract

It is often thought that the Qur'ān adopts a double and contradictory stance —at once for and against- Christianity. However, the Qur'ān does not only hold two types of texts of this nature, but rather four. How ought one to study such formulae? Did Muhammad seek the support of certain Christian groups, and later distance himself from them when they rejected his teachings? The present article develops a different hypothesis, namely that the formulae evince the gradual emergence of Islam between 610 and 710. It also explores the way in which Jesus was partially displaced by Muhammad as the new Messiah, and examines various texts suggesting that Muhammad, like the anonymous prophet of the Qur'ān , and formerly Jesus, was identified with the Son of Man of the Apocalyptic tradition.

\section{KeYwords}

Qur'ān; Christianity; Islam; Muhammad; Messiah; Apocalyptic Literature; the Son of Man.

1 El presente artículo no es sino la versión abreviada y simplificada del paper que presenté al primer Nangeroni Meeting del Early Islamic Studies Seminar, celebrado en Milán en junio de 2015; 



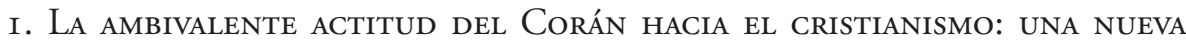
HIPÓTESIS

Como señalan acertadamente Marie-Thérèse Urvoy y Haggai Mazuz, el Corán despliega una actitud contradictoria — a la vez favorable y desfavorable — hacia el cristianismo ${ }^{2}$. Sin duda, la manera más sencilla e inmediata de interpretar este hecho consiste en pensar que Mahoma buscó el respaldo de ciertos grupos cristianos, y que más tarde se distanció de éstos al rechazar ellos sus enseńanzas ${ }^{3}$. Pero esta interpretación presenta varios problemas. Antes de examinarlos, sin embargo, me parece indispensable subrayar que los pasajes coránicos alusivos al cristianismo pueden y deben clasificarse de otra forma, ya que más bien que únicamente dos tipos de textos de esta índole (pro y anticristianos, respectivamente), el Corán contiene cuatro tipos de textos que conviene distinguir desde el primer momento y que podrían denominarse así:

(a) Fórmulas cristianas, a menudo con un cariz antijudío (e.g. Q 2:67-74?, $87,124,253 ; 4: 153-9 ; 5: 51$ ?, 70-1, 78-81?; 9:31?; 19:2-34a?; 23:49-50; 43:57-80?; 57:26-7?; 61:6?, 14);

(b) Fórmulas procristianas que ponen de relieve, por tanto, una cierta actitud de compromiso hacia el cristianismo (e.g. Q 3:42-64; 46-7, 78-81?, 826, 110-18?; 19:2-34a?; 43:57-80?; 57:26-7?);

véase https://www.academia.edu/3372907/A_Messianic_Controversy_Behind_the_Making_of_ Muhammad_as_the_Last_Prophet_2015_Conference_Paper_-_Upcoming_Book_Chapter. Agradezco a Daniel Beck, Gabriele Boccaccini, Michael Bonner, Emilio González Ferrín, Andreas Görke, Ghilène Hazem, Barbara Holcátová, Naomi Jacobs, Manfred Kropp, Ulrika Mårtensson, Haggai Mazuz, Alexander Mirkovic, Isaac Oliver, Cecilia Palombo, Jerome Parker, Anders Petersen, Karl-Friedrich Pohlmann, Meira Polliack, Parvaneh Pourshariati, Jan Retsö, Barbara Roggema, May Shaddel, Stephen Shoemaker, Francis Watson y, muy especialmente, Guillaume Dye, Claude Gilliot, David Powers, Gerd-R. Puin, Peter von Sivers y Philip Wood, sus comentarios y sugerencias.

2 M.-Th. Urvoy, "Chrétiens, Christianisme", en Dictionnaire du Coran, ed. M. A. Amir-Moezzi, 163-5 (París: Laffont, 2007); H. Mazuz, "Christians in the Qur'ān: Some Insights Derived from the Classical Exegetical Approach”, Studia Orientalia 112 (2012): 41-53.

3 D. Marshall, "Christianity in the Qur'ān", en Islamic Interpretations of Christianity, ed. Ll. Ridgeon (Londres y Nueva York: Routledge, $\left.{ }^{2} 2011\right), 16$. Cf. Mazuz, "Christians in the Qur'ān”, quien asume, en cambio, la interpretación tradicional según la cual hay que distinguir entre los cristianos que se convirtieron al islam y los que no lo hicieron, hacia cuyas creencias Mahoma se habría mostrado favorable y hostil, respectivamente. 
(c) Fórmulas de estilo polémico dirigidas contra ciertas creencias cristianas (e.g. Q 3:79-80; 4:171-2; 5:17, 72-7; 9:31?; 17:111; 19:34b-6; 112?); y

(d) Fórmulas sustitutorias cuyo objeto se diría que es remplazar al cristianismo y al judaísmo mediante algo nuevo y distinto (e.g. Q 3:65-88; 5:18-19, 44-7, 51?, 59, 65-9; 9:29-35; 19:88-95; 61:6?). ${ }^{4}$

Dicho de otro modo: lo que encontramos en el Corán, dependiendo del pasaje en cuestión que escojamos, oscila entre (1) la identificación con el cristianismo (lo que evidentemente implica mucho más que una mera actitud procristiana) = $a$, (2) una cierta actitud favorable hacia él $=b$, y una progresiva toma de distancia respecto del mismo que va desde (3) la controversia anticristiana (o la crítica a ciertas creencias propias de determinados grupos cristianos) $=c$ hasta (4) el rechazo y la sustitución del cristianismo (y del judaísmo) por otra cosa $=d$. Compárense, a título de muestra, los siguientes fragmentos:

(a) - Q 61:14:

“¡Oh, creyentes! ¡Sed auxiliares de Dios! [Actuad] como [cuando] Jesús, hijo de María, dijo a los apóstoles: ‘QQuién se une a mí para auxiliar a Dios?’ Los apóstoles dijeron: ‘ $¡$ Nosotros!’ . . .”.

(b) - Q 3:59-64:

" "59 Jesús es ante Dios semejante a Adán. Él le creó del polvo; le dijo: ‘Sé!', y fue. ${ }^{60} \mathrm{La}$ verdad [procede] de tu Señor, luego no te cuentes entre los que dudan. ${ }^{61} \mathrm{~A}$ quienes, tras haber recibido tú esta enseñanza, discutan contigo sobre esto, diles: 'Venid; llamemos a nuestros hijos y a vuestros hijos, a nuestras mujeres y a vuestras mujeres, a nosotros y a vosotros; imploremos a Dios e invoquemos su maldición sobre quienes mienten'. ${ }^{62}$ Ésta

4 Las interrogaciones indican que las fórmulas en cuestión podrían en realidad pertenecer a otro periodo. Todo depende en este punto de su interpretación. Así y por ejemplo, el texto estándar de Q 5:51 (“Oh, creyentes, no toméis a los judíos ni a los cristianos por amigos ...”) podría pertenecer al grupo $d$ más bien que al $a$; pero es posible interpretar la referencia a los "cristianos" como una interpolación (É.-M. Gallez, Le messie et son prophète. Aux origines de l'Islam [2 vols; Versalles: Éditions de Paris, 2005], 2:86-7) y situar dicho versículo, en consecuencia, dentro del grupo $a$. A su vez, Q 5:78 puede interpretarse como un texto sea cristiano, sea procristiano. Éste es también el caso de Q 5:110-18 y 57:26-7, mientras que Q 43:57-80 parece claramente cristiano en razón de su retórica particularmente enfática. Sobre Q 4:153-9, véase N. Robinson, Christ in Islam and Christianity: The Representation of Jesus in the Qur'an and the Classical Muslim Commentaries (London y Albany, NY: Macmillan y SUNY Press), 79-89, 106-11, 127-4; idem, "Jesus", en Encyclopaedia of the Qur'an, ed. J. D. McAuliffe (6 vols.; Leiden y Boston: Brill, 2001-6), 3:17-20; G. S. Reynolds, "The Muslim Jesus: Dead or Alive?" BSOAS 72.2: 237-58. Sobre Q 19:2-34, G. Dye, "Lieux saintes communs, partagés ou confisqués: aux sources de quelques péricopes coraniques (Q 19:16-33)", en Partage du sacré. Transferts, dévotions mixtes, rivalités interconfessionnelles, ed. I. Dépret y G. Dye (Bruselas: EME, 2012), 55-121. Volveré más adelante sobre Q 2:67-74; 9:31; 61:6 y 112. 
es la verdad: no hay más dios que Dios. Ciertamente, Dios es Poderoso, Sabio. ${ }^{63} \mathrm{Si}$ se apartan, [sabe que] Dios conoce a los corruptores. ${ }^{64} \mathrm{Di}$ [entonces]: 'Gente del Libro, ¡lleguemos a un acuerdo mutuo!, ¡[admitamos] que no adoramos sino a Dios, que nadie es similar a Él y que no tomamos por señores a otros que no sean Él!' Si se apartan [de vosotros], decid: ‘¡Damos fe de que nosotros nos sometemos [a Dios]!”’.

(c) $-\mathrm{Q} 4: 171:$

“¡Oh, gente del Libro! ¡ No ox excedáis en vuestra religión (o bien, no erréis en vuestro juicio $)^{5}$ ni digáis sobre Dios más que la verdad! El mesías, Jesús, hijo de María, no es sino el enviado de Dios y Su palabra, que él insufló en María, así como un espíritu procedente de Él. Creed en Dios y en sus enviados y no digáis ‘'Tres!' Desistid, es mejor para vosotros. Ciertamente, Dios es Uno; no le corresponde — iexaltado sea! — tener un hijo. ¡Suyo es cuanto hay en los cielos y en la tierra! ¡Dios basta como garante!”.

(d) Q 3:65-8:

${ }^{\text {"65 }}$ ¡Oh, gente del Libro! ¿Por qué discutís sobre Abraham cuando la Torá y el Evangelio fueron revelados después de él? ¿Es que no razonáis? ${ }^{66}$ Ved cómo sois: discutís sobre lo que sabéis, ¿pero por qué discutís sobre lo que no sabéis? ¡Dios sabe, mientras que vosotros no! ${ }^{67} \mathrm{Abraham}$ no era judío ni cristiano, sino que era un justo que se sometía [a Dios] y no se contaba entre los idólatras. ${ }^{68}$ Ciertamente, los más dignos de Abraham son quienes le siguen, el Profeta y los que creen [en su mensaje]. ¡Dios es el aliado de los creyentes!".

Dada la, a mi juicio, escasa fiabilidad de la denominada cronología tradicional del Corán - lo que vale también para su tímida adaptación por parte de Nöldeke, hoy demasiado fácilmente asumida como normativa ${ }^{6}-$, mi hipótesis es que $a, b, c$ y $d$ representan etapas sucesivas de lo que, a falta de otro término mejor, cabría llamar las relaciones tempranas entre el islam y el cristianismo (si es que es posible hablar del "islam" con anterioridad a $c$ o incluso d). Puede que determinadas polémicas concretas dieran paso a tales o cuales compromisos (en cuyo caso $c$, o algunos de los textos agrupados bajo $c$, podrían preceder a los incluidos en el apartado $b$, o al menos a algunos de entre éstos). Asimismo, es posible que

5 La primera lectura (lā taglū fì dìnikum) es la más habitual, pero la segunda (là ta'lī fì dìnikum) es igualmente posible, dada la ambigüedad del ductus consonántico $(\dot{\varepsilon} \mid \varepsilon=\varepsilon)$.

6 G. S. Reynolds, "Le problème de la chronologie du Coran”, Arabica 58 (2011): 477-502. Véase también G. Dye, "Pourquoi et comment se fait un texte canonique. Quelques réflexions sur l'histoire du Coran", en Hérésies: une construction d'identités religieuses, ed. Ch. Brouwer, G. Dye y A. van Rompaey (PHR. Bruselas: Éditions de l'Université de Bruxelles, 2015), 55-104. 
ciertas discrepancias cristológicas de las cuales el Corán se hace eco sean muy antiguas, y en rigor contemporáneas de los textos del grupo a (el estilo aparentemente arcaico de Q 112 da en sugerirlo así $\left.{ }^{7}\right)$. Por último, algunos de los textos del grupo $a$ podrían pertenecer más bien al grupo $d$ (cf. Q 61:5-6 en la vulgata uzmaní [“. . . Jesús, el hijo de María, dijo: 'Hijos de Israel, soy para vosotros un enviado de Dios que viene a confirmar lo que os fue dado antes en la Torá y que trae la buena nueva de otro enviado que vendrá después de mí, cuyo nombre es Aḥmad'... . ”] y en el códice de Ubayy b. Ka'b [“. . Jesús, el hijo de María, dijo: 'Hijos de Israel, soy para vosotros un enviado de Dios que trae la buena nueva de un profeta cuya comunidad será la última y por medio del cual Dios establecerá el sello de los profetas y los enviados'.... "], cuya referencia a una "nueva comunidad" cabe interpretar en clave sustitutoria). Pero creo que mi hipótesis ( $a>b$ $>c>d$ ) es también la más plausible y coherente, dado que se ajusta mejor que ninguna otra al esquema general característico de la formación transversal de las identidades religiosas en la Antigüedad tardía: (I) diseminación imprecisa de tales o cuales marcadores identitarios sobre un trasfondo de ideas y prácticas religiosas particularmente móvil y dinámico, (II) redistribución (y resemantización) de tales marcadores en función de una serie de líneas o ejes de cristalización creados ad hoc pero relativamente inestables aún, y (III) promoción y consolidación de estos últimos. Como he apuntado en otro lugar, "what usually begins as a juxtaposed set of indeterminate flows, gradually transforms into an agglomeration of interdependent clusters before narrowing into a few well-defined realms, be they ideas, communities, texts or practices"s.

Si mi hipótesis es correcta, por tanto, los pasajes cristianos (a), procristianos (b) y anticristianos $(c, d)$ contenidos en el Corán responden, grosso modo, a la siguiente distribución cronológica:

7 M. Kropp, "Tripartite, but Anti-Trinitarian Formulas in the Qur'ānic Corpus, Possibly PreQur'ānic”, en New Perspectives on the Qur'än: The Qur'än in Its Historical Context 2, ed. S. G. Reynolds (RSQ; Londres y Nueva York: Routledge, 2011), 247-64.

8 C. A. Segovia, The Quranic Noah and the Making of the Islamic Prophet: A Study of Intertextuality and Religious Identity Formation in Late Antiquity (JCIT 4; Berlín y Boston: De Gruyter, 2015), 8. 


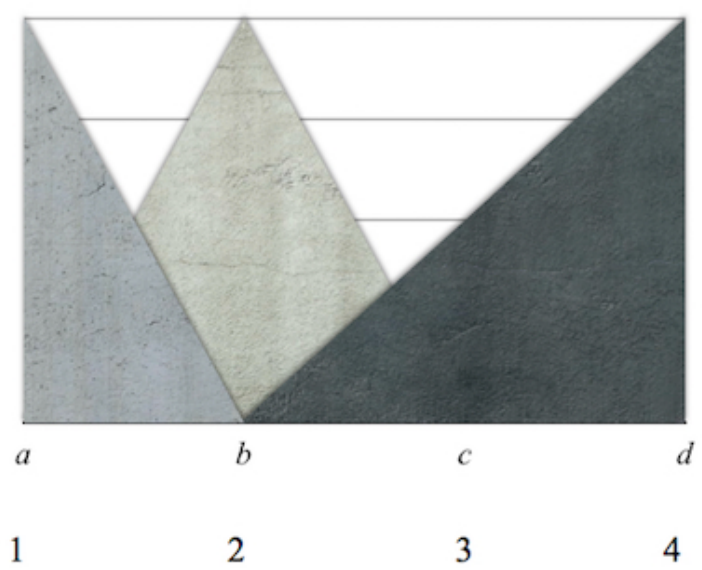

No pretendo con esto dar a entender que sea posible, ni mucho menos necesario, trazar una línea de demarcación exacta, esto es, una frontera clara y definitiva, entre las categorías $a, b, c$ y $d$, o entre los periodos $1,2,3$ y 4 , puesto que algunas de fórmulas aparentemente pertenecientes a uno de tales periodos podría eventualmente corresponder a otro; es más, diferentes tipos de fórmulas podrían caracterizar a un mismo periodo, ya que es difícil pensar que una única actitud prevaleciera en cada caso sobre las demás. Luego admito que el escenario sugerido más arriba no es el único posible. Pero, en términos generales, creo que es oportuno y legítimo trazar una línea divisoria entre esos cuatro periodos y categorías y que la idea fundamental que subyace a tal división —a saber, que el islam surgió de un medio originalmente cristiano- es plenamente válida. ${ }^{9}$

9 En un artículo de próxima publicación en M. Gross and K.-H. Ohlig, eds., Die Entstehung einer Weltreligion IV (Berlín: Hans Schiler, 2016), Peter von Sivers sugiere que el islam nació en época omeya y que sus raíces son cristológicas: "The most straightforward way to describe the formation of Islam is that it began with a christological debate within the Arab ruling class under Mu'āwiya, composed of generals, bureaucrats, scribes, and monks. In this debate, anti-Paschite and pro-Paschite, anti-Trisagion and pro-Trisagion convictions clashed. Traces of these clashes - which also involved polemics around Monophysite Julianism and tritheism - can be found in Penkāyē, Anastasius Sinaiticus, Arculf, and the Maronite Chronicle" (comunicación privada del 11 de julio de 2015). Mi hipótesis es distinta, pero debo reconocer que la suya es todo menos descabellada. Quisiera además expresarle aquí mi agradecimiento por compartir conmigo sus reflexiones antes de la publicación de dicho trabajo. 
2. El trasfondo histórico: de la destrucción del reino de Himyar a la CREACIÓN DEL ESTADO ÁRABO-ISLÁMICO EN ÉPOCA MARWANÍ

Analicemos ahora los problemas inherentes a la interpretación antes referida de la ambivalente actitud del Corán hacia el cristianismo, según la cual Mahoma habría buscado el apoyo de ciertos grupos cristianos, distanciándose luego de éstos al rechazar ellos sus enseñanzas.

El primer problema que presenta esta interpretación es — como ya he apuntado- que los textos más arriba agrupados bajo $a$ son plenamente cristianos más bien que procristianos; en otras palabras, expresan identificación con el cristianismo desde dentro más bien que una actitud favorable hacia el cristianismo desde fuera. Ahora bien, si admitimos que tales textos, o al menos la mayor parte de ellos, datan de la época de Mahoma y reflejan sus enseñanzas — cuyos oponentes, a juzgar por el cariz a un tiempo antijudío y antipagano de esos mismos textos, podrían haber sido entonces los politeístas y los judíos del Hiğāz, es decir, los dos grupos religiosos que se beneficiaron del declive del reino cristiano de Himyar en la segunda mitad del siglo VI_- uno debe preguntarse también en qué medida Mahoma pudo educarse en un medio cristiano y si su misión no consistió, al menos en un principio, en reafirmar una variante particular del cristianismo en su ciudad natal, La Meca ${ }^{10}$.

Se diría que las fuentes islámicas (Ibn Isḥāq, Ibn Sa'd, Ibn Hišām, al-Azraqī) conservan, aunque oblicuamente, cierta memoria de todo esto cuando señalan, por ejemplo, que la tumba de Jesús se encontraba cerca de Medina ${ }^{11}$, que no fue sino un monje cristiano llamado Bahịrā (al que los apologistas cristianos del siglo VIII califican de arriano) quien reconoció en Mahoma el signo de la profecía cuando este último no contaba más que nueve años, o que la actividad profética de Mahoma fue autentificada posteriormente por un sabio cristiano de La Meca, a saber: Waraqa b. Nawfal, o que Mahoma respetó los iconos de Jesús y María emplazados en el interior de la Ka'ba cuando conquisto La Meca en 630 y destruyó los ídolos contenidos en el santuario mequí. Véanse también los datos recopilados por Irfan Shahid ${ }^{12}$ sobre la existencia de varias iglesias (masăğid) dedicadas

10 Sobre la historia de la península arábiga entre los siglos IV y VII, véase G. Fisher, Between Empires: Romans, Arabs, and Sassanians in Late Antiquity (Oxford y Nueva York: Oxford University Press, 2011); Ch. J. Robin, "Arabia and Ethiopia", en The Oxford Handbook of Late Antiquity, ed. S. F. Johnson (Oxford y Nueva York: Oxford University Press, 2012), 247-332; G. W. Bowersock, Empires in Collision in Late Antiquity (Waltham, MA: Brandeis University Press e Historical Society of Israel, 2012); idem, The Throne of Adulis: Red Sea Wars on the Eve of Islam (Oxford y Nueva York: Oxford University Press, 2013).

11 G. D. Newby, The Making of the Last Prophet: A Reconstruction of the Earliest Biography of Muhammad (Columbia: University of South Carolina Press, 1989), 206, 211.

12 I. Shahid, Byzantium and the Arabs in the Fifth Century (Washington DC: Dumbarton Oaks, 1989), 333, 337-8, 345, 385-9, 390-2; idem, "Islam and Oriens Christianus: Makka 610-622 AD", en The Encounter of Eastern Christianity with Early Islam, ed. E. Grypeou, M. N. Swanson, y 
a María y un cementerio cristiano (maqbarat al-naṣārā) en La Meca en época preislámica, sobre un enclave próximo (¿quizá en la ruta hacia Nağrān?) conocido como mawqif al-nașrānì (la "parada de los cristianos") y sobre la posible conexión entre los Gurhum (a quienes suele atribuirse la introducción del cristianismo en el Hiğāa) y la (re)construcción de la Ka'ba.

Con todo, los indicios más interesantes acerca del trasfondo cristiano del primer islam son las descripciones coránicas de Jesús en tanto que Mesías (masīh) y Verbo divino (kalimatu llāhi) y en tanto que espíritu procedente de Dios (rūḥun minhu), títulos que no son aplicados a ningún otro personaje en el corpus coránico. Por su parte, Q 3:46 (wa yukallimu fi-l-mahdi) y 19:29-30 (kayfa nukallimu man kāna fì-l-mahdi șabiyyan) presentan implícitamente a Jesús como una figura suprahumana (¿a partir de 1 Henoc 106:1-3?). Mientras que Q 21:91 (fa-nafahnā fìhā min rrühināa) y 66:12 (fa-nafahnnā fìhi [sic!] min rrūhinina) sugieren que Jesús "participa" del Espíritu de Dios y que es a su imagen y semejanza en su calidad de segundo Adán; cf. Q 15:29; 38:72 (fa-id ā . . nafahtu fìhi min rrüḥ̂̀); Genesis 1:26-7; 2:7; y Hebreos 1:6. ${ }^{13}$

La pregunta que se impone en este punto es por tanto, a mi modo de ver, la siguiente: ¿propició el declive del reino cristiano Himyar por parte de los sasánidas hacia 565 -destrucción que permitió a los oponentes de este último, los politeístas y los judíos del sur y del oeste de la península arábiga, reconquistar el poder que dicho reino, fundado a finales del primer tercio del siglo VI, les había $\operatorname{arrebatado}^{14}$ _ , así como la subsiguiente invasión sasánida del Próximo Oriente cristiano en 610 (la fecha comúnmente asignada a las primeras revelaciones recibidas por Mahoma, que presentan rasgos apocalípticos muy marcados), propiciaron estos hechos — repito — una suerte de revival cristiano en el Hiğāz que pudo ser liderado, entre otros (sus profetas y profetisas rivales) por Mahoma? Michael Lecker y Glen Bowersock inciden en esto mismo desde otro ángulo, a mi juicio, cuando se preguntan si Mahoma pudo recibir en 622 (la fecha oficial de la hégira) el respaldo de Bizancio en su misión (cf. los pasajes aparentemente probizantinos contenidos en Q 18:83-102 y 30:1-3) ${ }^{15}$ ? Pues incluso si se con-

D. Thomas (Leiden y Boston: Brill, 2006), 12-13.

13 Es legítimo preguntar también si la "vaca" mencionada en Q 2:67-74, y que da su título al segundo capítulo del Corán, es una alusión a Cristo a partir de Barnabé 8:1-4 más bien que una referencia a Números 19 y/o Deuteronomio 21:1-9. O cuáles serían las implicaciones de leer almasīh en genitivo, en vez de en acusativo, en Q 9:31 ("Han tomado a sus rabinos y a sus monjes como señores aparte de Dios y del Mesías, el Hijo de María") - versículo al que tengo intención de dedicar un próximo trabajo.

14 Robin, "Arabia and Ethiopia", 297ss.

15 M. Lecker, "Were the Ghassānids and the Byzantines behind Muhammad's hijra?", en Les Jafnides. Des rois arabes au service de Byzance (VIe siècle de l'ère chrétienne), ed. Ch. J. Robin (París: De Boccard, de próxima publicación); Bowersock, Empires in Collision, 56. Sobre Q 18:83-102 véase K. Van Bladel, “The Alexander Legend in the Qur'ān 18:83-102”, en The Qur'ān in Its Histor- 
cede que los motivos de la conquista del Hiiğāz por parte de Mahoma entre 622 y 632 fueron ante todo políticos y han de examinarse a la luz de la contienda que enfrentó a los diferentes clanes árabes que pugnaban por alcanzar una nueva supremacía tribal en los territorios de la península arábiga tras la caída del reino de Himyar en 565 y la de los reinos gasánida y lájmida en 582 y 602; incluso si no podemos continuar pensando que el "islam" — que para entonces aún no existía - fue la causa principal de la conquista árabe del Próximo Oriente, es preciso, sin embargo, reconocer algún papel en ella al factor religioso; y comprender que la alianza política y religiosa entre los árabes y los imperios de los que ellos habían sido vasallos durante siglos (el romano, el bizantino y el sasánida) nunca significó la completa sumisión de los árabes respecto de sus señores políticos, ya que tales imperios dejaron siempre cierto margen de maniobra a sus aliados, tanto en lo administrativo como en lo religioso ${ }^{16}$.

El segundo problema que plantea la interpretación antes mencionada de la ambivalente actitud del Corán hacia el cristianismo — según la cual Mahoma habría buscado el apoyo de ciertos grupos cristianos, distanciándose luego de éstos al rechazar ellos sus enseñanzas - es que apunta a que las fórmulas agrupadas en el epígrafe $c$ (es decir, las fórmulas polémicas dirigidas contra la creencia de que Jesús no sólo sería el mesías, sino también el Hijo de Dios, y contra la interpretación triteísta de la trinidad) representarían una plena ruptura con el cristianismo. Es interesante reparar, en este sentido, en una serie de inscripciones sudarábigas de mediados del siglo VI, debidas al rey cristiano del origen etiópico Abraha (CIH 541, DAI GDN 2002-20 y Ry 506 ), en cuya invocación preliminar son mencionados "el Misericordioso (rhmnn) y su Mesías ( $\left.m s^{l} h-h w\right)$ " (o bien ambos acompañados del Espíritu Santo $[r h q d s])$ en vez de "el Padre y el Hijo" ${ }^{17}$. En mi opinión, esto indica que los cristianos del sur de la península arábiga veían a Jesús como el Mesías, pero no necesariamente como el Hijo de Dios. Y desafía por tanto la opinión, muy extendida pero incorrecta, de que, en los albores del islam, los árabes cristianos del sur de la península —al igual que sus vecinos cris-

ical Context, ed. G. S. Reynolds (RSQ; Londres y Nueva York: Routledge, 2008), 175-203; T. Tesei, "The Prophecy of Dū-l-Qarnayn and the Origins of the Qur'ānic Corpus", Miscellanea Arabica (2013-14): 273-90.. Sobre Q 30:1-3, Bowersock, Empires in Collision, 60-77.

16 G. Fisher, "Between Constantinople and Ctesiphon: Arab tribes, Arab leaders, and religious identity in late antique Syria, Arabia, and Mesopotamia", workshop paper, Universidad de California en Santa Bárbara, https://lists.lsit.ucsb.edu/archives/borderlands.religion/attachments/20130530/93f0d24c/attachment-0001.pdf.

17 C. A. Segovia, "The Jews and Christians of pre-Islamic Yemen (Himyar) and the Elusive Matrix of the Qur'ān's Christology", paper presentado a la 8th Annual ASMEA Conference, Washington, DC, 29-31 octubre 2015, https://www.academia.edu/14840043/The_Jews_and_Christians_of_pre-Islamic_Yemen_Himyar_and_the_Elusive_Matrix_of_the_Qur_āns_Christology_2015_Conference_Paper; idem, "Abraha's Christological Formula Rhmnn $w$-Ms ${ }^{I} h-h w$ and Its Relevance for the Study of Islam's origins”, Oriens Christianum (de próxima publicación). 
tianos, árabes o no, de Siria-Palestina - eran mayormente miafisitas y defendían, así pues, una cristología alta. ${ }^{18}$ En fin, el hecho de que esa misma fórmula ("alRaḥman" + "Jesús el Mesías") sea repetida una y otra vez en el Corán es sumamente elocuente. Por su parte, los cristianos dyofisitas de Iraq y de la franja oriental de la península arábiga — a quienes el Libro de los Himyaríes y la Crónica de Seert presentan como fundadores del más importante de los centros cristianos del sur de la misma: Nağrān - aceptaban, al igual que sus rivales miafisitas, la divinidad de Jesús, pero, como es sabido, acentuaban, frente a éstos, su naturaleza específicamente humana ${ }^{19}$.

¿Pueden entonces las fórmulas anticristianas del Corán definirse realmente así? ¿Por qué no verlas más bien como fórmulas expresa o tácitamente dirigidas contra la cristología miafisita en general o contra sus variantes julianista (que ponía aún mayor énfasis en la divinidad de Jesús) y filoponiana (que daba en interpretar la trinidad en clave triteísta)? Por otra parte, y dado que el cristianismo miafisita era la corriente predominante en Siria-Palestina (y Egipto), ¿qué nos impide tomar dicha región más bien que el Hiğāz, y por ende el periodo comprendido entre 632 y 692 - o sea, entre la fecha generalmente asignada a las primeras incursiones árabes en Siria-Palestina y la fecha en la que fueron puestos precisamente allí los cimientos del nuevo estado árabo-islámico_-, como su escenario más plausible y verosímil?

18 Cf. las inscripciones de Esimifaios, antecesor inmediato de Abraha, las cuales aluden a Jesús en tanto que "Hijo de Dios" [ $b n-h w]$, y la basmala etiópica tradicional: "En el nombre del Padre, del hijo (wäld) y del Espíritu Santo" (M. Kropp, "»Im Namen Gottes, (d. i.) des gnädigen (und) B/ (b)armherzigen«. Die muslimische Basmala: Neue Ansätze zu ihrer Erklärung”, Oriens Christianus 97 [2013-14]: 195). El hecho de que fuera en tiempos del emperador miafisita Anastasio I Dicoro — tal y como narra Juan Diacrinomeno en su Historia eclesiástica — cuando el cristianismo adquirió forma institucional en el sur de la península arábiga, y de que las persecuciones anticristianas en la región alcanzaran su cenit hacia 518 con el asesinato del obispo miafisita de Nağrān y la masacre de numerosos cristianos miafisitas en dicha localidad en 523, ha llevado a numerosos investigadores a dar sin más por sentado que el cristianismo miafisita representaba la opción cristiana dominante en la zona. Pero, en rigor, no puede afirmarse más que coexistían allí diferentes grupos cristianos, sin que sea posible determinar el porcentaje de la población que integraba cada uno.

19 El nombre de Jesús en el Corán (acerca del cual véase G. Dye y M. Kropp, "Le nom de Jésus ('İsā) dans le Coran, et quelques autres noms bibliques: remarques sur l'onomastique coranique", en Figures bibliques en islam, ed. G. Dye y F. Nobilio (Religion et Alterité; Fernelmont: EME, 2011, 171-98) y su denominación en tanto que "el Mesías, hijo de María" (cf. la mención de María en tanto que Christotókos en vez de Theotókos, común entre los dyofisitas) evocan la cristológía siríaco-oriental. Repárese también en las referencias positivas a la religión de los árabes que ofrecen diversos autores dyofisitas de mediados del siglo VII: Išō yahb III's en sus cartas 48B (97) y 14C (251), el autor de la Crónica de Juzistán (34) y Juan bar Penkāyē en sus Cuestiones fundamentales (141); véase a este respecto M. Ph. Penn, When Christians First Met Muslims: A Sourcebook of the Earliest Syriac Writings on Islam (Oakland, CA: California University Press, 2015), 33, 36, 50, 889. Sobre los orígenes presumiblemente dyofisitas del Islam, véase Segovia, "Jews and Christians"; idem, "Abraha’s Christological Formula". 
Esto nos lleva al tercero de los problemas inherentes a la interpretación de la ambivalente actitud del Corán hacia el cristianismo como exponente del acercamiento inicial de Mahoma a ciertos grupos cristianos y su posterior rechazo de sus creencias. Cuatro de los textos polémicos incluidos en el epígrafe $c$, a saber: Q 4:171-2; 17:111; 19:34b-6; 112, figuran en la inscripción de la cara interior de la arcada octogonal de la Cúpula de la Roca en Jerusalén, erigida por el califa 'Abd al-Malik b. Marwān en 692 y en la cual encontramos también, por primera vez, la palabra "islam" —así como el nombre de "Mahoma" asociado de algún modo a esa noción ${ }^{20}$. ¿Refleja tal inscripción, aunque con leves variaciones, el texto coránico? Ésta es la interpretación más común, pero no es la única posible ni tampoco, a mi juicio, la más convincente. Pues, sencillamente, no disponemos de ningún manuscrito coránico anterior al califato de 'Abd al-Malik, y no podemos datar con seguridad los fragmentos coránicos más tempranos que tenemos antes de ese periodo. ${ }^{21}$ Dicho de otro modo: los textos de la Cúpula de la Roca podrían haber sido encargados para el edificio y haber sido, en consecuencia, redactados en 692 o un poco antes. Al fin y al cabo, es en la Cúpula de la Roca - erigida como emblema del nuevo estado árabo-islámico creado por 'Abd al-Malikdonde los hallamos por primera vez ${ }^{22}$. Pero entonces podría ser también que los

20 A menos que se entienda que $m h m(m) d$ no funciona ahí sino como un participio pasivo con valor gerundivo empleado para designar a Jesús (Ch. Luxenberg, "A New Interpretation of the Arabic Inscription in Jerusalem's Dome of the Rock", en The Hidden Origins of Islam, ed. K.-H. Ohlig y G.-R. Puin [Amherst, NY: Prometheus Books, 2010], 125-51), lo cual es posible pero, en mi opinión, improbable (cf. P. von Sivers, "Christology and Prophetology in the Umayyad Arab Empire”, en Die Entstehung einer Weltreligion III, ed. M. Gross y K.-H. Ohlig [Berlin: Hans Schiler, 2014], 255-85; C. A. Segovia, "Identity Politics and Scholarship in the Study of Islamic Origins: The Inscriptions on the Dome of the Rock as a Test Case", en Identity, Politics, and Scholarship: The Study of Islam and the Study of Religions, ed. M. Sheedy [CE; Sheffield y Bristol, CT: Equinox, de próxima publicación]). Sobre la política "muhammadiana" de 'Abd al-Malik y su contribución a la creación del hasta ese momento inexistente estado árabo-islámico, véase Ch. F. Robinson, 'Abd al-Malik (Oxford: Oneworld, 2005). Sobre los fragmentos de la Cúpula de la Roca y su posible trasfondo ideológico, Van der Velden, "Die Felsendominschrift als Ende einer christologischen Konvergenztextökumene im Koran", Oriens Christianus 95 (2011): 213-46; Segovia, "Identity Politics".

21 La datación de BNF Arabe 328 hacia la década de 670, propuesta for François Déroche (La transmission écrite du Coran dans les débuts de l'islam. Le codex Parisino-petropolitanus [Leiden y Boston: Brill, 2009]; idem, Qur'ans of the Umayyads [LSIS 1; Leiden y Boston: Brill, 2014]), obedece a criterios paleográficos; es por tanto necesario someter dicho manuscrito — por otra parte fragmentario - a análisis químico. En cuanto al palimpsesto de Șanăa’, su análisis químico ha dado resultados desiguales, cuando no extravagantes (388-588, 433-599, 543-643 y 578-669), lo que impide determinar su fecha de composición. Y ante los fragmentos de Tubinga y Leiden conviene mostrarse cautos: su análisis químico da una como resultado horquilla temporal de seis décadas (650-710), pero también una franja cronológica adicional que no puede descartarse (750-760), y cabe además preguntarse de qué tipo de documento proceden. En suma, la pretensión de que la vulgata uzmaní dataría de mediados del siglo VII carece de fundamento histórico.

22 Cf. A.-L. de Prémare, "'Abd al-Malik b. Marwān and the Process of the Qur'ān's Composition", en The Hidden Origins of Islam: New research into Its Early History, ed. K.-H. Ohlig y G.-R. 
textos agrupados en los epígrafes $a$ y $b$ los precedieran en el tiempo y que los incluidos en el epígrafe $d$ fueran posteriores a ellos.

Teniendo todo esto en cuenta, me atrevo a proponer la siguiente cronología, que permitiría afianzar y concretar el esquema evolutivo presentado en la primera sección de este trabajo:

- Grupo $a$ = Estadio 1: Fórmulas muhammadianas tempranas;

- Grupo $b$ = Estadio 2: Fórmulas muhammadianas tardías o, alternativamente, postmuhammadianas —en cuyo caso quizá entonces premarwaníes, es decir, contemporáneas de la conquista árabe del Próximo Oriente (¿y de Mu'āwiya?);

- Grupo $c=$ Estadio 3: Fórmulas postmuhammadianas, posiblemente marwaníes (¿de época de 'Abd al-Malik?);

- Grupo $d$ = Estadio 4: Fórmulas postmuhammadianas, posiblemente también marwaníes pero posteriores a las del grupo anterior (¿de época de Walìd b. 'Abd al-Malik?).

En resumen, tendríamos, primero $(1 / a)$, una serie de textos que reflejarían una fe aún básicamente cristiana y que podrían datarse hacia la década de 610 (¿y 620 ?); a continuación (2/b), una serie de textos que reflejarían, en cambio, una actitud procristiana y que podrían ser contemporáneos de la expansión árabe de los años 630-80 - personalmente yo me inclinaría por datarlos en tiempos de Mu āwiya, dado su acentuado sentido de la diplomacia (Robinson 2005: 24-5), lo que implicaría que al menos una parte de las fórmulas incluidas en el epígrafe $b$ habrían sido redactadas entre 661 y 680 ; y, por último $(3 / c, 4 / d)$, una serie de textos que reflejarían la formación progresiva de la nueva identidad religiosa promovida por 'Abd al-Malik b. Marwān entre 692 y 705 (3/c) y consolidada por su hijo y sucesor Walīd I entre 705 y 715 (4/d). ${ }^{23}$.

Puin (Amherst, NY: Prometheus Books, 2010), 193.

23 Repárese en el carácter aún ambiguo de la retórica que exhiben, pese a todo, las inscripciones de la Cúpula de la Roca, en las que no acaba de saberse quién es el "enviado" y el "siervo de Dios" (¿Jesús o Mahoma?; Segovia 2016a), y en el hecho de que "Abd al-Malik todavía acuñara monedas con el signo de la cruz acompañando a la šahāda (T. Ramadan, "A Rare Arab-Byzantine Hybrid Coin of Damascus: An Intriguing 'Mule' Bearing a Standing Emperor Obverse and a Sphere through Pole-on-Steps Reverse”, JONS 203 [2010]: 43-5). Cf. Y. D. Nevo y J. Kohen, Crossroads to Islam: The Origins of the Arab Religion and the Arab State (Amherst, NY: Prometheus Books, 2003), 271-95; Robinson, Abd al-Malik, 59-128; F. M. Donner, Muhammad and the Believers: At the Origins of Islam (Cambridge, MS: Harvard University Press, 2010), 194-224. Mi intención es ampliar este enfoque en un futuro libro que estoy escribiendo con mi colega de la Universidad Libre de Bruselas ULB, Guillaume Dye, cuyo título provisional es Re-Imagining Islam in the Late 7th 


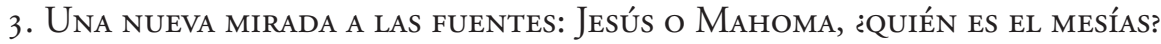

Varios de los textos incluidos en $a, b$, y $c$ (Q 3:45; 4:157, 171-2; 5:17, 72, 75), y al menos uno de los incluidos en $d(\mathrm{Q}$ 9:30), sostienen que Jesús es el mesías. He ahí un interesante indicio, en mi opinión, de que la mesianidad de Jesús fue perdiendo relevancia para los seguidores de Mahoma hacia la década de 710. Nadie, sin embargo, parece haberse percatado de ello hasta aquí.

A mediados del siglo VIII, Ibn Isḥāq describe a Jesús como un profeta que adquirió naturaleza angélica tras su ascenso celeste ${ }^{24}$. Y ya sea el propio Ibn Ishậ, o Ibn Hišām cerca de un siglo después, confieren a Mahoma una serie de rasgos noáquicos y cristológicos que se diría que lo transforman en un personaje no ya carismático, sino también mesiánico (¿quizá en sustitución de Jesús?). He examinado esta cuestión en otro lugar ${ }^{25}$, por lo que no creo necesario volver aquí sobre ella. Baste con señalar que Ibn Isḥāq/Ibn Hišām narra(n) que un ángel anunció a la madre de Mahoma el futuro nacimiento de su hijo, que una luz emanaba de ella durante su embarazo y que del padre de Mahoma, antes de tomarla como esposa, brotaba asimismo una misteriosa luz, como si la milagrosa simiente de Mahoma aguardara un seno en el que fructificar (Sīra 100-2, ed. Wüstenfeld ${ }^{26}$ ). Todo esto recuerda el nacimiento luminoso de Noé, Melquisedeq y Jesús en los diferentes textos parabíblicos que he analizado en mi trabajo sobre el Noé parabíblico ${ }^{27}$ y, de nuevo, mi libro sobre el Noé coránico. Repárese, además, en que Mahoma es calificado de "señor" S 101, mientras que en S 171 leemos que es similar a una "palmera de fructíferas ramas" (una metáfora comúnmente empleada en el judaísmo para aludir a la simiente de los justos) y que ha venido para "separar al hombre de su padre, o de su hermano, o de su mujer, o de su familia" (cf. Mateo 10:34-6). Estos elocuentes motivos refuerzan mi impresión de que Mahoma es implícitamente presentado como un mesías por Ibn Isḥāq/Ibn Hišām ${ }^{28}$. Cf. no obstante la subordinación de Mahoma a Jesús en S 106, así como el modo en que Jesús es cuestionado en $S 237$ por haberse convertido en objeto de culto entre sus seguidores. Así pues, el estatuto mesiánico de Mahoma en Ibn Isḥāq/Ibn Hišām es ambiguo. Pero quizá esto sea normal, pues quizá llegó un momento en el que

Century. Un avance de sus contenidos podrá consultarse en https://www.academia.edu/7050551/ Re-Imagining_Islam_in_the_Late_7th_Century_with_Guillaume_Dye_2017_Upcoming_Book).

La cronología propuesta en el presente trabajo es sin embargo mía, por lo que asumo también la responsabilidad de sus posibles errores.

24 Newby, Making of the Last Prophet, 206.

25 Segovia, Quranic Noah, 102-7.

26 F. Wüstenfeld, Das Leben Muhammeds nach Muhammed Ibn Ishâk bearbeitet von Abd el-Malik Ibn Hischâm (3 vols; Gotinga: Dieterich, 1858-60).

27 C. A. Segovia, "Noah as Eschatological Mediator Transposed: From 2 Enoch 71-72 to the Christological Echoes of 1 Enoch 106:3 in the Qur'ān" Henoch 33.1 (2011): 130-45.

28 Cf. J. M. F. van Reeth, "La typologie tu prophète selon le Coran: le cas de Jésus", en Figures bibliques en Islam, ed. G. Dye y F. Nobilio (Fernelmont: EME, 2011), 81-105. 
resultó más fácil para los seguidores de Mahoma apoyarse en una nueva figura carismática desprovista de los rasgos divinos atribuidos a Jesús por la mayoría de los cristianos, que continuar proclamando la mesianidad de este último; lo que no les obligaba, sin embargo, a renunciar a su papel escatológico y soteriológico, que fue transferido a Mahoma en su calidad de "último profeta"29.

Otros indicios de la interpretación de Mahoma como un nuevo mesías pueden encontrarse también en los textos estudiados por Uri Rubin en 1975, a los que sorprendentemente - casi nadie ha prestado renovada atención desde entonces ${ }^{30}$.

Además de ampliar el motivo de la simiente milagrosa de Mahoma y de su cuerpo luminoso, estos textos afirman (i) que la luz de Mahoma (o "luz muhammadiana”) existía ya antes de que fuera creado el trono de Dios y, por lo tanto, antes de la creación de los cielos, la tierra y el mar; y (ii) que el nombre de Mahoma estaba inscrito desde el primer momento $\left(i i_{1}\right)$ en el trono de Dios (al que confería además estabilidad), $\left(i{ }_{2}\right)$ sobre los hombros de Adán (así como también en las hojas de los árboles del paraíso, entre los ojos de los ángeles y en el cuello de las huríes), y que ( $i_{3}$ ) era conocido por los ángeles, los demonios, los profetas, los habitantes del paraíso y del infierno, los continentes y los océanos.

Ejemplos de ii se encuentran en al-Bayhaqī, Dalāil al-Nubuwwa $\left(b_{1}\right)$; al-Halabī, al-Sìra al-Halabiyya $\left(i i_{1}, i i_{2}\right)$; al-Hुarkūšī, Šaraf al-Nabiyy $\left(i i_{1}, i i_{2}\right)$; Ibn al-Ğaw-

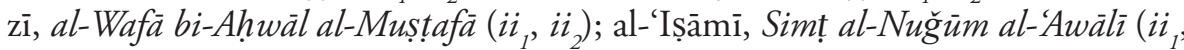

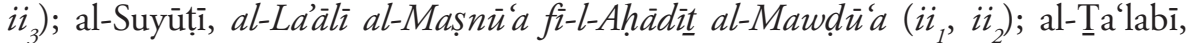
Qiṣaș al-Anbiyā' (ii $\left.{ }_{2}\right)$; y al-Zurqānī, Šarh 'alà al-Mawāhib al-Laduniyya li-l-Qastallāni $\left(i i_{1}, i i_{2}\right)$. Rubin subraya con razón que estos textos de proveniencia tanto sunní como chií se hacen eco de la descripción del mesías Hijo del Hombre en 1 Henoc 48:3, donde se menciona que el nombre de este último fue pronunciado ante el Señor de los Espíritus (es decir, ante Dios) antes de que el sol y las constelaciones fueran creados. Pero Rubin se detiene ahí. Es sin duda preciso notar que el versículo precedente (48:2) y los vv. 48:6 y 62:7 contienen afirmaciones parecidas, señalándose además en ellos que el Hijo del Hombre fue elegido al principio de los tiempos y ocultado por Dios junto a sí en el esplendor de su poder antes de crear el mundo; y que estos pasajes del Libro de las Parábolas se basan en la elec-

29 Sobre Mahoma como el último de los profetas, véase D. S. Powers, Muhammad Is Not the Father of Any of Your Men: The Making of the Last Prophet (DRLAR; Filadelfia: University of Pennsylvania Press, 2009), idem, Zayd (DRLAR; Filadelfia: University of Pennsylvania Press, 2014). Sobre Mahoma como profeta escatológico, S. J. Shoemaker, The Death of a Prophet: The End of Muhammad's Life and the Beginnings of Islam (DRLAR; Filadelfia: University of Pennsylvania Press, 2012), 118-96; Segovia, Quranic Noah. Por su parte, K. Szilágyi, "A Prophet like Jesus: Christians and Muslims Debating Muhammad's Death", JSAI 36 (2009): 131-71, analiza diversas tradiciones tempranas según las cuales Mahoma, al morir, ascendió -al igual que Jesús- a los cielos.

30 U. Rubin, "Pre-Existence and Light: Aspects of the Concept of Nūr Muhammad", IOS 5 (1975): 62-119. 
ción del profeta "desde el seno" materno en Isaías 49:1, la creación de los ángeles en Jubileos 3:2-11 y el papel creador asignado a la Sabiduría en Proverbios 8:2231 y Sirácida 24:1-3, mientras que la noción del que el mesías oculto se revelará al final de los tiempos deriva a su vez de 4 Esdras 13:26, 52.

Por su parte, $i$ se divide en dos submotivos bien ilustrados en las obras anteriormente citadas de al-Hुarkūšī, Ibn al-Ğawzī, al-'Ișāmī, al-Suyūṭī, al-Ta'labī y al-Zurqānī, así como en al-Mas'ūdī, It bāt al-Wașiyya li-l-Imām 'Alī b. Abì Ṭalib, donde leemos $\left(i_{1}\right)$ que la "luz muhammadiana" fue la razón por la que Dios creó a la humanidad, los cielos, la tierra, el paraíso y el infierno; y $\left(i_{2}\right)$ que ella no es únicamente la causa, sino también la sustancia de la creación divina. Al-Zurqānī traza un riguroso paralelismo entre Jesús y Mahoma en este sentido, mientras alHarkūšš, curiosamente, relaciona la idea de que con Mahoma todo comenzó con la noción de que él es el "sello" de los profetas.

Pero quizá el pasaje más fascinante de todos sea el reproducido en al-Harkūšī, Šaraf al-Nabiyy; Ibn Šahrāšūb, Manāqib Āl Abì Ṭālib; al-'Ișāmī, Simṭ al-Nuğūm al-Awālī; y al-Mağlisī, Biḥār al-Anwār, donde lemmas que "cuando Halīma (la comadrona de Mahoma) lo tomó entre sus brazos él abrió los ojos y éstos refulgían.” Esta narración completa, añadiéndole su otra mitad, la que encontramos acerca de Jesús en Q 3:46; 19:29-30: “Él [= Jesús] hablará a los hombres desde la cuna y [lo mismo hará] cuando sea adulto, y se contará entre los justos"; "Ella [= María] le señaló. Pero ellos dijeron: ‘Cómo vamos a hablar con alguien que aún está en la cuna, con un recién nacido?' Él [= Jesús] dijo entonces: 'En verdad yo soy el siervo de Dios. Él me ha dado el Libro y ha hecho de mí un profeta". Cf. 1 Hen 106:23, donde esta misma historia y el símbolo mesiánico que ella contiene (relativo al recién nacido cuyos ojos refulgen y es capaz de hablar desde la cuna) figuran en su integridad: "Cuando él [= Noé] abrió los ojos la casa brilló como el sol. Entonces se incorporó de entre las manos de la comadrona, abrió su boca y alabó al Señor".

Así pues, la suposición de que Mahoma únicamente fue visto como un profeta por todos sus seguidores es errónea, pues hubo también, al parecer, quienes le vieron como un nuevo mesías. En líneas generales, puede decirse que la identificación de Mahoma con el mesías conoció probablemente tres fases: en un principio, quien fue identificado con el mesías fue más bien Jesús; luego, los rasgos mesiánicos de este último fueron transferidos a Mahoma, aunque de manera nunca declarada; finalmente, tal identificación se rechazó, viéndose sustituida por la noción de que Mahoma constituía, simplemente, el "sello" de los profetas, por lo que debía vérsele también como el más eminente de entre todos ellos. Ésta es, justamente, la creencia mayoritaria del islam. Con todo, y como acabamos de ver, algunos de sus rasgos mesiánicos lograron sobrevivir en diferentes círculos a lo largo de toda la Edad Media. La "biografía” de Mahoma de Ibn Isḥāq/Ibn Hišām pertenece a la segunda de esas fases, mientras que las obras antes mencionadas de

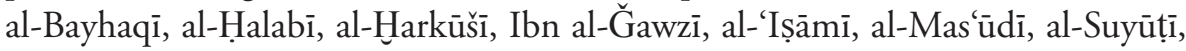


al-Ta 'labī y al-Zurqānī reflejan la posteridad de la imagen mesiánica de Mahoma en época medieval —y aún con posterioridad a ésta ${ }^{31}$.

4. Sobre la identificación del profeta coránico con el mesías Hijo del Hombre: a propósito de Corán i i:35, 49, Lucas i7:26, Mateo 24:37-9, IsAÍAS 54:7-IO Y 4QTANĤ̂MÎM 9-I 3

¿Pero qué ocurriría si el propio profeta anónimo del Corán — cuya identificación con Mahoma es menos segura de lo que suele pensarse, dado que su nombre no nos es suministrado salvo en cuatro ocasiones susceptibles de ser consideradas interpolaciones posteriores; y si es que puede hablarse, por otra parte, de un único profeta coránico, lo cual es igualmente discutible (Segovia 2015b: [140-1])—, qué ocurriría si el profeta anónimo del Corán hubiera sido identificado en algún momento, también él, con el mesías? Ésta es por lo general una cuestión asimismo soslayada, pero muy importante, puesto que una eventual respuesta afirmativa implicaría que la identificación de Mahoma con el mesías no representó ningún tipo de innovación en realidad.

Hay algo sumamente curioso en este sentido en las palabras de consolación dirigidas al profeta coránico en Q 11:35, 49 — versículos que, a mi juicio, fueron tardíamente incorporado al corpus coránico (Segovia 2015b: 67-8, 70-84). Tales versículos siguen una pauta retórica sorprendentemente similar a la que encontramos en 4QTanhûmîm 9-13, un manuscrito fragmentario en hebreo hallado en la Cueva 4 de Qumrán en el que las palabras de consolación dirigidas por Dios a Noé en Génesis 8:21-2; 9:8-17 son — siguiendo la pauta suministrada en Isaías 54:7-10 - empleadas para confortar al hablante en sus tribulaciones ${ }^{32}$. La identificación tipológica de los tiempos de Noé con el final de los tiempos (cf. 1 Henoc 93:4), y por tanto con la era mesiánica en la que se espera la venida del Hijo del Hombre es explícita en Mateo 24:37-9, Lucas 17:26 y 2 Pedro 3:5-7,1012, e implícita en la identificación del mesías con el propio Noé. Pero entre todos los escritos judíos y cristianos anteriores al Corán, 4QTanhûmîm es único en su identificación del hablante con Noé y en la formulación de tal identificación en mitad de un discurso divino — ilos dos motivos que encontramos de nuevo en Q 11:49!

La aparente relación entre Q 11:35, 49, Is 54:7-10 y 4QTanḥûmîm 9-13 puede ser casual o no. Si se trata de una simple coincidencia, cabe únicamente

31 Cf. la defensa de Mahoma en tanto que Mesías verdadero (frente a Jesús) en Juan Alonso Aragonés y el Evangelio de Bernabé (G. A. Wiegers, "Mahoma visto como el Mesías: comparación de las obras polémicas de Juan Alonso con el Evangelio de Bernabé [1]", ILU 1 [1996]: 197-222; idem, "Mahoma visto como el Mesías: comparación de las obras polémicas de Juan Alonso con el Evangelio de Bernabé [2]", ILU 2 [1997]: 199-228).

32 Cf. D. M. Peters, Noah Traditions in the Dead Sea Scrolls: Conversations and Controversies of Antiquity (EJL 26; Atlanta, GA: SBL, 2008), 134-5. 
entonces inferir que el autor o los autores de Q 11:35, 49 emplearon sin saberlo - luego por azar - un modelo retórico ya existente, con todo, para enfatizar así el nexo simbólico susceptible de ser trazado entre los tiempos de Noé y el fin de los tiempos de la apocalíptica. Si no se trata, en cambio, de una mera coincidencia, se perfilan entonces tres posibilidades:

$(\alpha)$ el autor o los autores de Q 11:35, 49 tomaron como referente Is 54:710 y/o 4QTanhûmîm 9-13 (o un documento similar hoy perdido), pero no los pasajes neotestamentarios anteriormente mencionados, no siendo su intención otra que la de subrayar que el profeta coránico era, por decirlo así, el heraldo del éschaton y, en consecuencia, la persona llamada a preparar la venida más o menos inminente del mesías;

$(\beta)$ el autor o los autores de Q 11:35, 49 tomaron como referente no sólo Is 54:7-10 y/o 4QTanhûmîm 9-13 (o un documento similar hoy perdido), sino también los pasajes neotestamentarios citados, pero únicamente con intención de subrayar, una vez más, que el profeta coránico era el heraldo del éschaton;

$(\gamma)$ el autor o los autores de Q 11:35, 49 veían al profeta coránico como el Hijo del Hombre y querían ponerlo así de manifiesto.

Ninguna de estas hipótesis puede descartarse de antemano. Por otra parte, la naturaleza compuesta del corpus coránico y el hecho — ya apuntado- de que tras los diferentes escritos que conforman este último podría haber varios más bien que un único profeta, permiten pensar que incluso si el profeta aludido en $\mathrm{Q}$ 11:35, 49 fue visto por sus seguidores como el mesías, los demás profetas anónimos del Corán no tendrían por qué caer bajo esa identificación. Finalmente,

$(\delta)$ sería posible interpretar Q 11:35, 49 como una interpolación tardía tendente a proyectar la condición mesiánica de Mahoma sobre el profeta anónimo del Corán (o, como mínimo, sobre uno de los diferentes profetas aludidos en él, según lo ya indicado).

Como quiera que sea, tales hipótesis refuerzan, de un lado, la idea de que el corpus coránico fue redactado, o al menos compilado, en un medio de escribas, $y$, de otro lado, la idea de que la mesianidad de Mahoma es un asunto que debe examinarse aún con el detalle necesario a fin de comprender mejor la identidad atribuida al profeta del islam en la Antigüedad y las complejas relaciones existentes entre las primitivas creencias islámicas y la fe cristiana de la que el islam fue desprendiéndose gradualmente — si bien quizá más tarde y de manera infinitamente más ambigua de lo que suele suponerse- antes de llegar a ser la nueva religión con la que hoy lo asociamos. 